\title{
Kinetics of Oil Dispersion in the Absence and Presence of Block Copolymers
}

\author{
H. Polat \\ Dept. of Chemistry, I zmir Institute of Technology, G aziosmanpasa Bulv. N o. 16, 35230 C ankaya, I zmir-T urkey \\ M. Polat \\ M ining E ngineering D ept., D okuz E ylül U niversity, 35100 B ornova-I zmir, T urkey \\ S. Chander \\ D ept. of E nergy and Geo-E nvironmental Engineering, The Pennsylvania State U niversity, 115 H osler B Idg., \\ U niversity Park, PA 16802
}

\begin{abstract}
A phenomenological model proposed describes droplet breakup in the turbulently agitated lean oil-in-water dispersions and provides a correlation between the median droplet size in an agitated $v$ essel of standard geometry and the time of dispersion. It was assumed that the droplet breakup takes place in the dispersion-only region and coalescence is negligible. The model described the data from this study and the literature quite satisfactorily under these conditions. The effect of adding triblock PEO/PPO/PEO copolymeric surfactants on the dispersion kinetics of oil was also investigated. Addition of surfactant reduced the median oil droplet size significantly, and the extent of this reduction was a strong function of surfactant concentration. Application of the model on these data demonstrated that the change in the median droplet size could be divided into two distinct regions. The breakage rate was high initially, most probably due to continuous adsorption of surfactant molecules at the oil/water interface. A lower breakage rate was attained at longer times, as the surfactant molecules were depleted from the solution. The time of transition between the two was affected strongly by the concentration of the surfactant added. Furthermore, the time of addition of the surfactant did not affect the final droplet-size distribution in the system.
\end{abstract}

\section{Introduction}

Dispersion of oil into fine droplets is important in many applications, such as flotation, selective agglomeration, solvent extraction, wastewater treatment, and oil drilling. Size distribution of oil droplets determines the rate of mass transfer between the continuous and the disperse phase and the outcome of the process in these applications.

Breakup of the droplets is usually achieved by applying mechanical energy in the form of agitation, which deforms the interface between the dispersed and continuous phases. Change in the size distribution of oil droplets in an agitated vessel with time depends on two subprocesses: dispersion and coalescence. Either of these subprocesses can dominate, de-

Correspondence concerning this article should be addressed to $\mathrm{H}$. Polat. pending on the agitator speed, oil concentration, and the additives present in the system. Surface-active additives, that is, surfactants, affect these subprocesses by:

- Decreasing the oil/water interfacial tension. Surface active agents that adsorb at the oil/water interface are known to decrease the interfacial tension significantly even at very low concentrations. The nonionic copolymeric surfactants employed in this study cause a decrease in the surface tension of about 20-30 dyne/cm at concentrations below $10^{-6}$ $M$. A reduction in the restoring surface-tension force against deformative stresses such as those induced by eddies at turbulent mixing promotes the dispersion of oil.

- Creating a double layer at the oil/water interface. Oil droplets dispersed in water can develop a surface charge de- 
pending on solution chemistry (A damson, 1990; H iemenz, 1986), which leads to the development of an electrical double layer. The presence of such a layer affects the interaction of two oil droplets electrostatically. Adsorbed species are expected to alter the degree of interaction between the droplets by influencing the profile of the double layer.

- Influencing film drainage. Coalescence of two oil droplets is only possible if the water film between the two can drain away during the period of contact. The presence of surfactant molecules or colloidal particles within the water film can change the energy required for drainage, and hence the rate of coalescence, depending on the degree of hydration of these species (Nikolov and Wasan, 1989; Polat and Chander, 1994).

- Causing steric interaction. Depending on the type of surfactant and the adsorption mechanism, surfactant molecules adsorbed at the oil/water interface may have hydrated sections extending outward from each droplet surface. When two such droplets approach each other, the extended regions of the surfactant molecules physically interact, preventing further approach for successful coalescence (steric stabilization).

A knowledge of the size of the droplets in a unit process is important for modeling and control. Several studies have been conducted to obtain mathematical models for predicting the droplet-size distribution, either under equilibrium conditions or as a function of agitation time. H inze (1955) suggested that a drop would break if the ratio of the deforming external stress and the counteracting internal stress due to the interfacial tension, $\gamma$, reached a critical value. Shinnar (1961) used the $\mathrm{H}$ inze approach to develop an empirical dispersion model to obtain

$$
\mathrm{X}_{\max }=\mathrm{D}_{\mathrm{a}} \mathrm{CW} \mathrm{e}^{-0.6}
$$

where $X_{\max }$ is the maximum droplet size, $D_{a}$ is the impeller diameter, $C$ is a constant, and We is the $W$ eber number, which is defined as

$$
\mathrm{We}=\rho \mathrm{N}^{2} \mathrm{D}_{\mathrm{a}}^{3} \gamma^{-1}
$$

where $\mathrm{N}$ is the impeller speed, and $\rho$ is the density of the continuous phase. Sprow (1967) also suggested the preceding expression and adduced that the value of the constant was between 0.126 and 0.15. Coulaloglou and Tavlarides (1976) discussed various correlations based on Eq. 1 that are available in the literature. A rai et al. (1977) and Konno et al. (1982) argued that the viscous properties of the dispersed phase that were neglected in the model proposed by Shinnar (1961) were important with regard to the droplet size. A model that took the viscous properties of the dispersed phase into account was proposed by Lagisetty et al. (1986). Koshy et al. (1988), who maintained that all these models overpredict the maximum stable drop size in the presence of surfactants, developed a model incorporating the dynamic and static interfacial tensions to account for the presence of the surfactant molecules in solution.

Other approaches have also been used to estimate the droplet-size distribution in an agitated vessel. Narsimhan et al. (1980) utilized the experimental measurements of drop distributions along with a population balance approach to determine the probability of breakage rate based on the similarity concept in lean dispersions. Nishikawa et al. (1990) assumed that the drop-size distribution in a vessel was determined by the mutual relation between the energy dissipation rate, the residence time of drops at a certain location, the breakup rate of drops, and the coalescence rate of drops, and suggested correlative equations that represented the drop-size distribution under various mixing conditions. In addition, computer simulations of the emulsification process under turbulent flow conditions were carried out by several investigators (Becher and McCann, 1991; Tjaberinga et al., 1993; Lachaise et al., 1995).

In this study, a setup was designed to carry out in situ size-distribution measurements as a function of time using light scattering. A phenomenological model for lean dispersion systems was developed to predict the median droplet size as a function of time. The model allows the kinetics of dispersion to be described by two parameters: the median droplet size after $1 \mathrm{~min}$ of dispersion and a dimensionless breakage rate constant. The effect of the addition of block copolymeric surfactants on the kinetics of dispersion of oil was also investigated.

\section{Method}

Several experimental techniques have been utilized in the literature to determine the droplet-size distributions. These include:

- Photography (Coulaloglou and Tavlarides, 1976)

- Microscopy (Lagisetty et al., 1986; Koshy et al., 1988; Nishikawa et al., 1990)

- Light scattering (Polat and Chander, 1992; Chander et al., 1994)

- High-speed camera recordings (Polat and Chander, 1992)

- Electronic counting (Sachs and Rushton, 1954; Vermeulen et al., 1955; Calderbank, 1958; Sleicher, 1962; Sprow, 1967).

A size-distribution measurement could be carried out either by batch or in situ. U sually a small sample is obtained from the vessel and subjected to size measurement in the batch measurements. This method may not yield the actual size distribution in the vessel because the droplets coalesce, since a finite time elapses between the sampling and the measurement. Encapsulation of oil droplets by chemical means has been practiced to prevent coalescence ( $M$ adden and M cCoy, 1964; M lynek and Resnick, 1972; Narsimhan et al., 1980; Lagisetty et al., 1986). Encapsulation "freezes" the system by creating a polymeric film around the droplets such that the size distribution is affected minimally by the changes in the agitation conditions. The fact that it is difficult to carry out kinetics experiments is a drawback of the encapsulation procedure. A given kinetics test must be repeated for each time interval, since the chemistry of the system changes upon encapsulation. It was also found by $\mathrm{N}$ arsimhan et al. (1980) that the polymeric film around the droplet was stronger for small drops, which means that large encapsulated drops are more susceptible to breakage during sampling. M ore importantly, the encapsulation procedure presents complications 


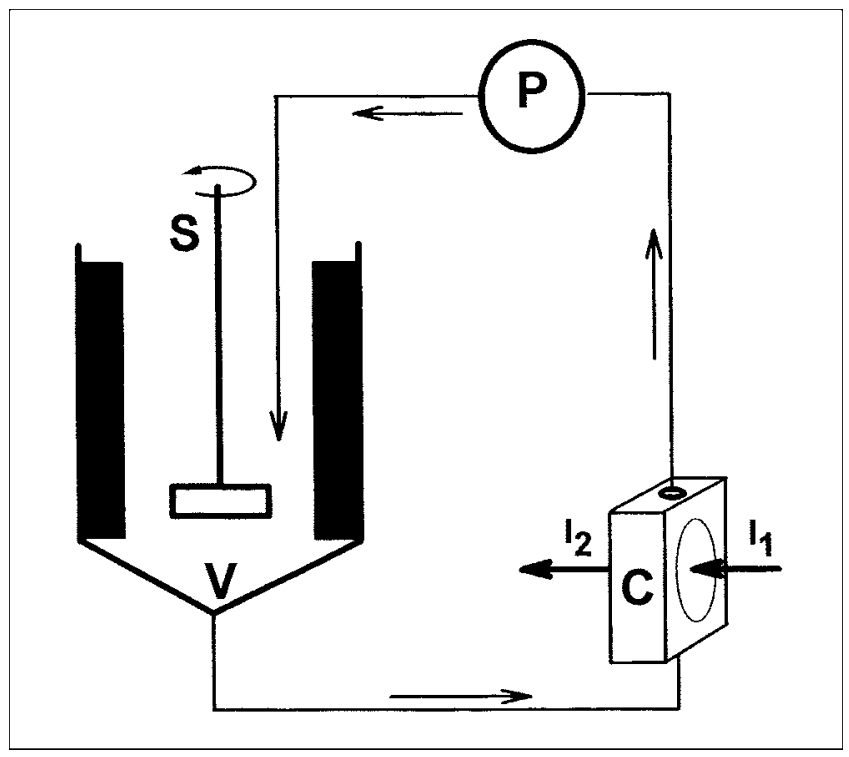

Figure 1. Experimental setup used in the study.

V: agitated vessel; S: stirrer; $C$ : flowthrough cell of the size measurement device; $P$ : peristaltic pump; $I_{1}, I_{2}$ : the lights coming from the laser source and going to the detector, respectively.

when the effect of other reagents, such as surfactants, on the dispersion behavior of oil is to be determined due to the interactions of the encapsulating chemicals with these reagents. On the other hand, in situ measurements of the size distribution in a vessel is hampered by many factors, depending on the method utilized. For example, high-speed filming, photography, and microscopy of the oil droplets suffer from the small number of observable droplets because of the limited depth of field. Similarly, the light-scattering methods cannot be utilized in measuring the droplet-size distribution directly in the dispersion vessel due to the limitations in the length of the laser beam's path. In addition, the location of the measurement point influences the apparent size distribution. The setup designed in this study to carry out in situ size-distribution measurements as a function of time using light scattering is believed to minimize the drawbacks of this method.

\section{Setup}

The setup (Figure 1) contained a 2-liter vessel of standard geometry (H olland and Chapman, 1966), with four baffles and a turbine-type stirrer (Figure 2). The agitation speed was maintained constant at $1000 \mathrm{rpm}$ to within $1 \%$ of the nominal value using a feedback-looped constant-torque drive. The vessel was connected to a flowthrough cell with a 10-in.-long 1/8-in.-diam. M asterflex tubing. The flowthrough cell was placed in the light beam path of a light-scattering size measurement device, Malvern M odel 2600c, for in situ size measurements. An adjustable-speed peristaltic pump was placed after the measurement cell to circulate the solution. A known amount of dodecane was added to the agitated vessel in the form of a pulse input, and the time of addition was recorded as the zero time of dispersion. Size-distribution measurements were obtained at preset time-intervals.

\section{Calibration}

In order to confirm that the in situ size distributions obtained using the setup given in Figure 1 corresponded to the actual size distribution of the oil droplets in the agitated vessel at that time, the following experiment was conducted. A $n$ oil-soluble monomer (terephaloyl chloride) was dissolved in the oil phase prior to the experiment. Monomer concentration was $0.05 \%$ by volume of the amount of oil in the system. The oil-plus monomer mixture $(0.1 \%$ by volume of the total solution volume in the vessel) was introduced and dispersion was initiated at an impeller speed of $1000 \mathrm{rpm}$ while the solution was circulated through the system. A fter 16 minutes of agitation, the size distribution of the droplets was measured while the solution containing the droplets was passing through the flowthrough cell using the light-scattering device. A s soon as the size measurement was over, a water-soluble monomer (piperazine, $0.05 \%$ by volume based on the amount of water in the system) was added to the agitated vessel. Following the addition of piperazine the agitation speed was reduced to 200 rpm, which was enough to keep the droplets suspended but not so intense as to cause further dispersion. Addition of piperazine resulted in an encapsulating polymeric film around each droplet due to the reaction of piperazine with terephaloyl chloride at the oil/water interface. This encapsulation effectively froze the dispersion process. F urther details of the encapsulation process are given elsewhere (M lynek and R esnick, 1972). A $n$ aliquot of sample taken from the agitated vessel was diluted and transferred into a batch-measurement cell with a gentle, built-in agitation mechanism. The light-

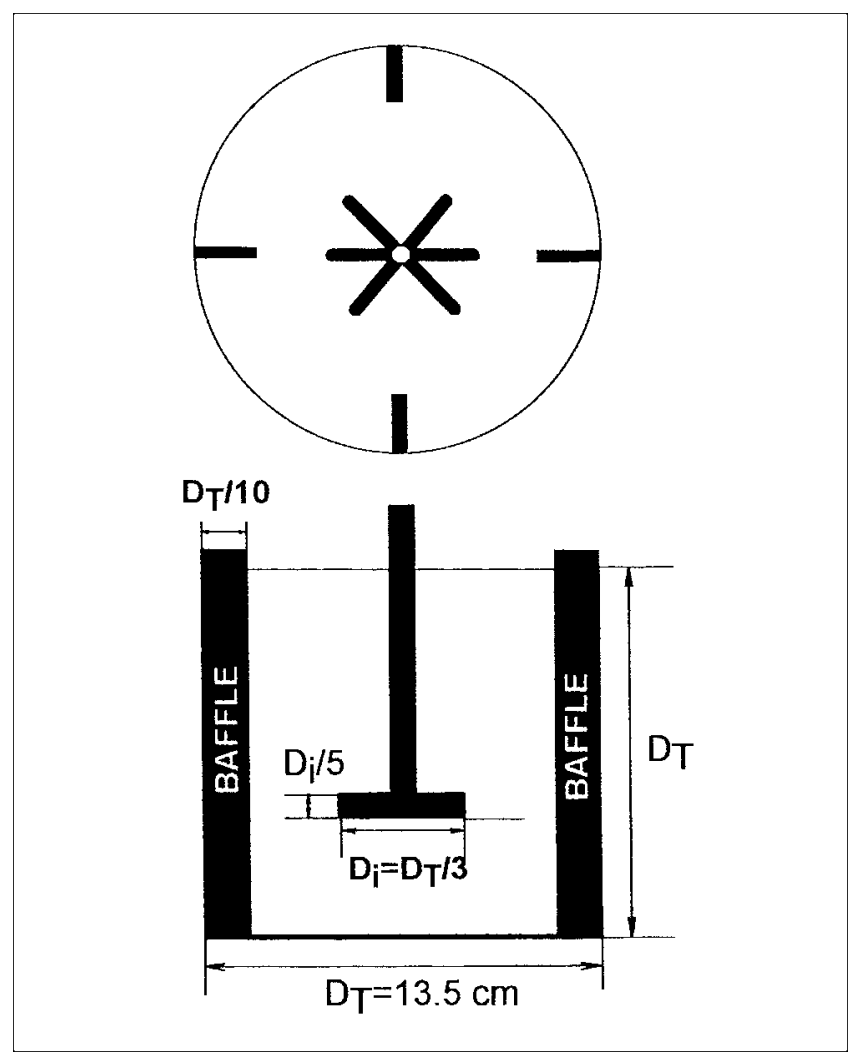

Figure 2. Agitation vessel and the turbine-type stirrer used in this study. 


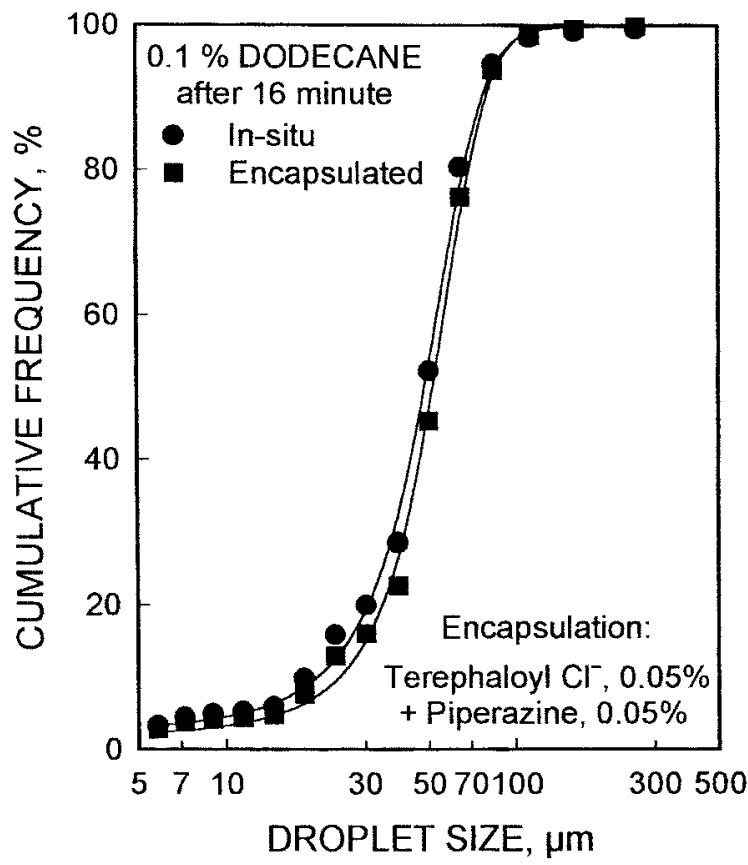

Figure 3. Size distribution of oil droplets measured in-situ in the flow through vessel and after encapsulation using piperazine and terephaloyl chloride.

scattering device was later used to measure the size distribution of the encapsulated droplets in this batch cell. Figure 3 gives the two size distributions, one in situ while the solution containing the actual oil droplets was passing through the flowthrough vessel, and the other from the batch measurement with the encapsulated oil droplets. The figure shows that the two size distributions are quite similar. This demonstrates that the size distributions, which were measured in situ by the flowthrough vessel, indeed represent the actual size distribution in the agitated vessel.

\section{Material}

Pluronic surfactants used in this study, namely Pluronics L-64 and P-104, were obtained from BASF Corporation, Washington, NJ. These surfactants, which were in the form $\mathrm{PEO/PPO/PEO} \mathrm{block} \mathrm{copolymers,} \mathrm{were} \mathrm{water} \mathrm{soluble} \mathrm{and}$ were used as aqueous solutions in the concentration range of $10^{-9}$ to $10^{-4} \mathrm{~mol} / \mathrm{L}$. Detailed information about these reagents is presented elsewhere (Polat, 1995; Polat and Chander, 1999).

Reagent-grade $n$-dodecane from Aldrich was used in the experiments. Double-distilled water with a resistivity of at least $1.5 \mathrm{M} \Omega$ was utilized to prepare the surfactant solutions. The glassware used was cleaned with a chromic acid solution.

\section{Phenomenological Dispersion Model}

The droplet-size distributions for the dispersion of $0.1 \%$ of dodecane in water obtained by the setup described earlier are given in Figure 4a as a function of agitation time. The normalized size distributions for the same data presented in Figure $4 \mathrm{~b}$ demonstrate that the size distributions are self-pre- serving. Thus, the median droplet size $\left(\mathrm{X}_{50}\right)$ was utilized to represent the changes in the drop size as a function of time. The time dependence of the median droplet size, $X_{50}$, for the data in Figure 4 is given in Figure 5, along with some additional measurements to show the reproducibility of the experiment.

The dispersion model was based on the study of Hinze (1955), who suggested that breakage of an isolated droplet is determined mainly by the ratio of external and internal stresses acting on such a droplet. The external stress, $\tau$, is the force per unit surface area and acts in such a way as to cause deformation on the droplet. H owever, the interfacial tension, $\gamma$, will give rise to a surface force that will counteract the deformation. If $X$ is the diameter of the droplet, the internal stress due to the surface tension force will be of the order of magnitude $[\gamma / \mathrm{X}]$. Hinze suggested that the probability of breakage would be related to a generalized Weber number, which is defined as the ratio of external and internal stresses such that

$$
\mathrm{We}=\frac{\tau \mathrm{X}}{\gamma} .
$$

$H$ inze stated that a larger We number meant a larger external force, $\tau$, compared with the counteracting interfacial tension-force $\gamma / \mathrm{X}$. This, in turn, would cause greater deformation and, at a critical value (We) crit, $_{\text {, breakup would occur. }}$ This approach was used by other investigators to develop an empirical dispersion model to predict the maximum stable

\section{DROPLET SIZE, $\mu \mathrm{m}$}

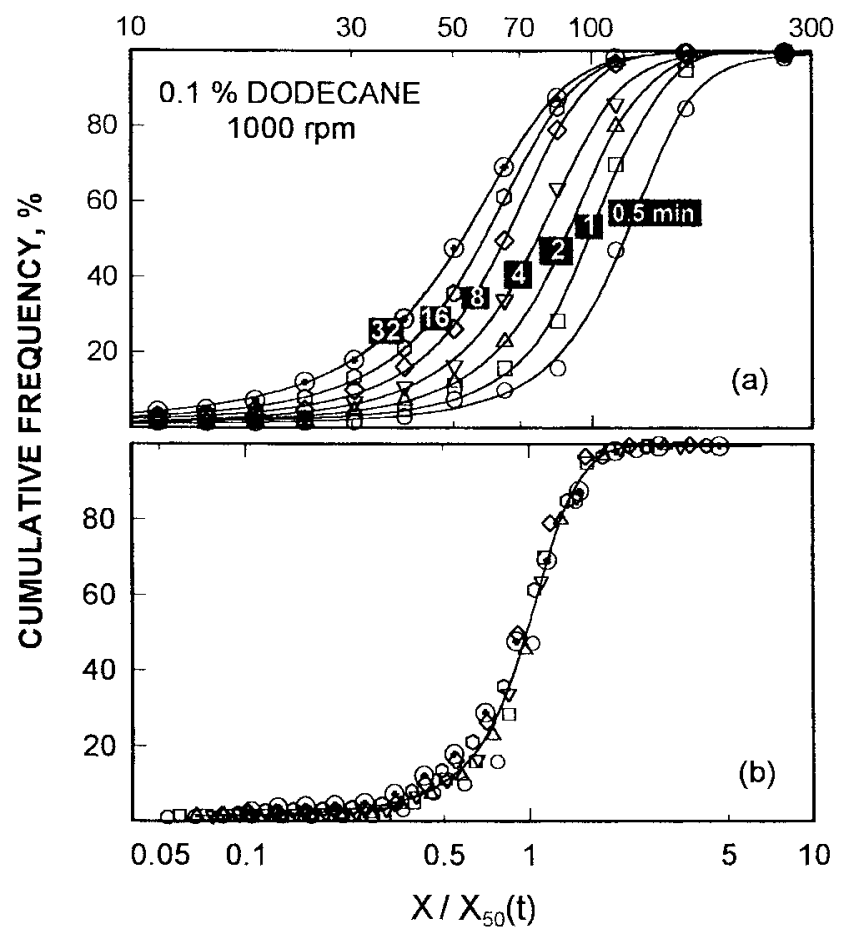

Figure 4. (a) Size distribution of dodecane droplets in water at various times; (b) normalized size distribution of dodecane droplets in water. 


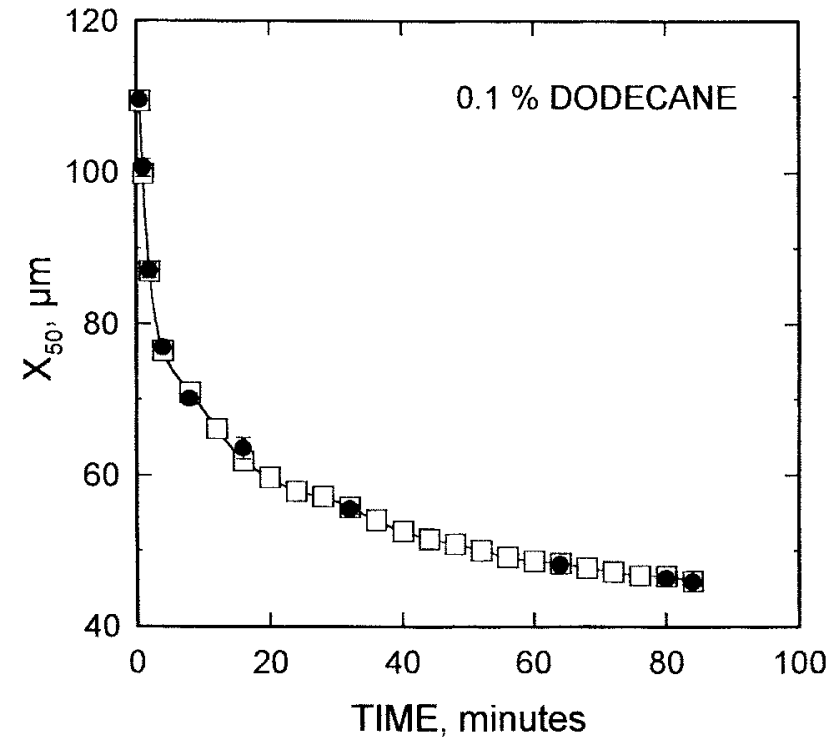

Figure 5. Median droplet size, $X_{50}$, as a function of agitation time for $0.1 \%$ dodecane.

Filled symbols represent the results of an identical experiment to show the reproducibility. The brackets on the symbols at 1,2, and 16 min give the $95 \%$ confidence interval for 7-repeat experiments.

droplet diameter, $X_{\max }$, under steady-state conditions (Shinnar, 1961; Sprow, 1967; Coulaloglou and Tavlarides, 1976; L agisetty et al., 1986; Koshy et al., 1988).

In a standard vessel with four baffles and an agitation speed at $1000 \mathrm{rpm}$, the flow regime is turbulent. In such a system there will be a distribution of eddies of various sizes (W alstra, 1983) that induce the external stresses that lead to deformation of droplets. Glasgow and H su (1985) suggested that the efficiency of breakage was a function of both the size of the eddies and the droplets; thus, a practical limit for particle-size reduction in a given system was a length scale on the order of the Kolmogorov's microscale of turbulence.

If the energy input into the dispersion vessel is constant, it is reasonable to assume that the distribution of the eddy sizes will be relatively stable, resulting in a time-invariant microscale of turbulence. Nevertheless, the average droplet size will gradually decrease with time. If the microscale of turbulence is comparable to the droplet size, a significant and increasing fraction of droplets with sizes less than the microscale of turbulence will be generated during the dispersion process, decreasing the breakage rate as a function of time. Hence, it could be suggested that the rate of change in the droplet size would be inversely proportional to the dispersion time. The functional form, which is not known, could be estimated from actual dispersion experiments. A fter calculating the Kolmogorov'smicroscale of turbulence, $\lambda_{0}$, for the system, one could experimentally determine the fraction of droplets that are finer than $\lambda_{0}, F\left(\lambda_{0}, t\right)$, as a function of time. The functional relationship between the breakage rate and the dispersion time can be estimated from a plot of $F\left(\lambda_{0}, t\right)$ vs. t. This exercise has been carried out for our system as follows.

By dimensional analysis, Kolmogorov (1949) suggested that the dissipation rate, $\epsilon$, and kinematic viscosity, $\nu$, can be arranged to give a length-scale for the turbulence, $\lambda_{0}$, in the system:

$$
\lambda_{0}=\left(\frac{\nu^{3}}{\epsilon}\right)^{1 / 4} \quad \text { and } \quad \epsilon=\frac{\mathrm{P}}{\mathrm{m}}
$$

where $\nu$ is the kinematic viscosity, $\mathrm{P}$ is the power input into the system, and $m$ is the mass of the medium in the tank. Power input is given by

$$
\mathrm{P}=\mathrm{N}_{\mathrm{p}} \mathrm{D}_{\mathrm{a}}^{5} \mathrm{~N}^{3} \rho,
$$

where $N_{p}$ is the power number, which is a function of the R eynolds number. For the standard four-baffled vessel with a turbine-type stirrer operated at 1,000 rpm, the Reynolds number is on the order of $10^{5}$, which results in a turbulent flow regime. In this region $N_{p}$ is constant and equal to 4 for the vessel geometry employed (Tatterson, 1991). This yields a power input of about $1.025 \mathrm{~W}$, which in turn gives a $\lambda_{0}$ value of about $30 \mu \mathrm{m}$.

The change in the fraction of droplets that are finer than $\lambda_{0}=30 \mu \mathrm{m}$ as a function of time is given in Figure 6 . The figure shows that the fraction of droplets that fall below the microscale of turbulence increases linearly with time, with the exception of very short times. This demonstrates that the breakage rate will be inversely proportional to the first power of time.

The phenomenological model proposed is based on H inze's criterion and the inverse relationship between the breakage rate and the time of dispersion. If breakage is dominant, the change in the median droplet size with time will be proportional to the generalized We number defined by $\mathrm{H}$ inze, while

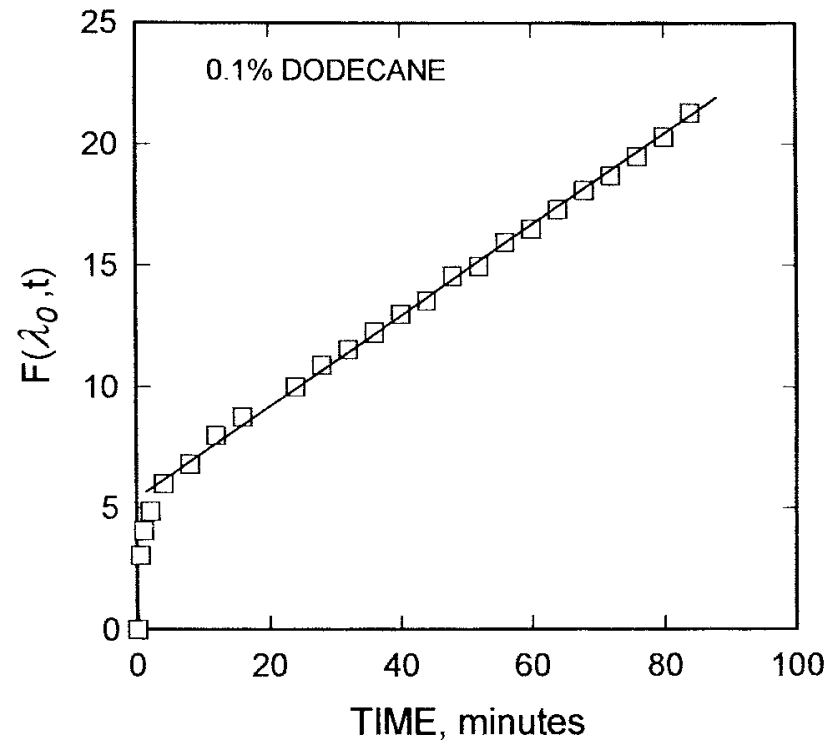

Figure 6. Fraction of droplets that are smaller than the Kolmogorov's microscale of turbulence, $\lambda_{0}$, as a function of agitation time. 
it will be inversely proportional to dispersion time. That is,

$$
\frac{d}{d t}\left[X_{50}(t)\right]=-k^{\prime}\left[\frac{\tau(t) X_{50}(t)}{\gamma}\right] \frac{1}{t},
$$

where $\mathrm{X}_{50}(\mathrm{t})$ and $\tau(\mathrm{t})$ are the median droplet size and the external stress per unit area at time t, respectively; $\gamma$ is the interfacial tension; and $\mathrm{k}^{\prime}$ is a proportionality constant. Assuming that $\tau(\mathrm{t})$ can be replaced with a time-averaged stress per unit area, $\tau$ (Tatterson, 1991), $\mathrm{k}^{\prime}, \tau(\mathrm{t})$, and $\gamma$ could be collected as a new, dimensionless constant $\mathrm{k}$ and the preceding equation could be integrated to give

$$
\ln \left[X_{50}(t)\right]=\ln \left[X_{50}(1)\right]-k \ln (t),
$$

where $X_{50}(1)$ is the median droplet size at one minute of dispersion.

A plot of $\operatorname{In}\left[X_{50}(t)\right]$ vs. In $(t)$ should result in a straight line with an intercept of $\ln \left[X_{50}(t=1)\right]$ and a slope of $k$. A plot of the dispersion data previously given in Figure 5 is presented in Figure 7 on a log-log scale. Both these data and the data from $\mathrm{N}$ arsinhan et al. (1980), given as an inset in Figure 7 , support the validity of the model.

\section{Effect of Dodecane Concentration}

While the model represented the kinetics data quite well for a dodecane concentration of $0.1 \%$, the behavior of the system was somewhat different when the dispersion was carried out at a dodecane concentration of $1.0 \%$ by volume. The median droplet sizes as a function of agitation time are given

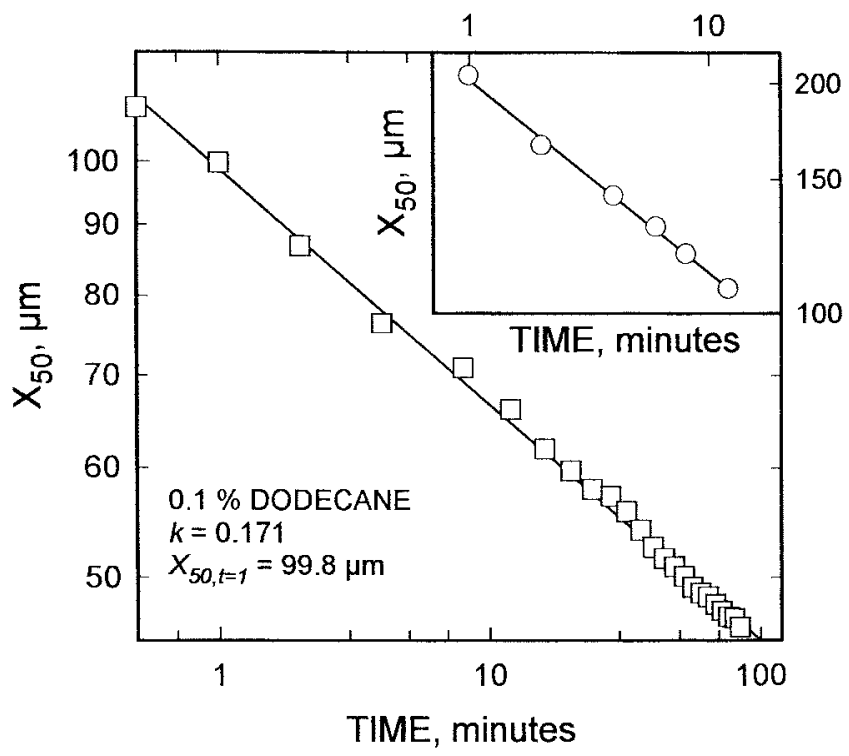

Figure 7. Median droplet sizes as a function of agitation time; symbols: experimentally determined solid line: predicted by Eq. 8.

The figure inset on the top right shows the test of the model with the data from $\mathrm{N}$ arsimhan et al. (1980) $\left[\mathrm{CCl}_{4}+\right.$ 0 ctane (50-50\% ) in water; 3-paddles; $30^{\circ} \mathrm{C}$; $\left.420 \mathrm{rpm}\right]$.

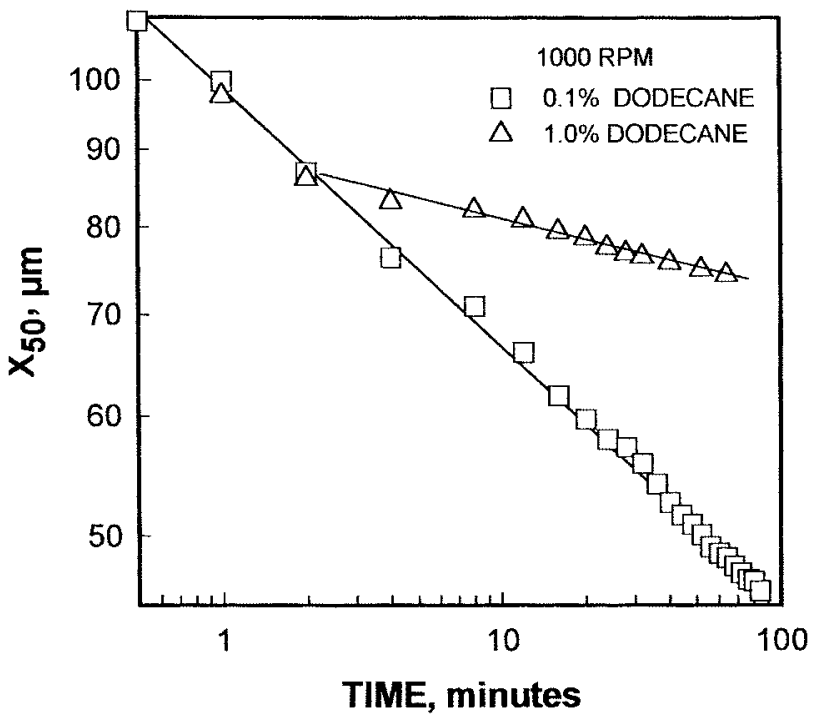

Figure 8. Change in the median droplet size as a function of time at dodecane concentrations of $0.1 \%$ and $1.0 \%$ by volume.

for $1.0 \%$, along with the $0.1 \%$ data in Figure 8 . The data for the $1.0 \%$ case do not seem to follow a straight line for the full time scale as required by the model, but consist of two separate straight lines. These data are able to show, however, that there is an initial period of about 2 minutes where dispersion is the dominant mechanism as expected, but coalescence starts becoming effective afterwards. The slope of the curve is different for these two regions; it is high in the dispersion-dominated region, resulting in a rapid decrease in the median droplet size, and becomes lower as coalescence increases, resulting in a slower decrease in the median droplet size.

\section{Effect of Stirrer Speed}

In order to see the effect of concentrations at various stirrer speeds, the model was applied to the data from Polat and Chander (1992). The results given in Figure 9 demonstrate that a straight line is obtained at a stirrer speed of $1,400 \mathrm{rpm}$ up to a dodecane concentration of $1.0 \%$. A lso, dispersion studies with a dodecane concentration of $1.0 \%$ at impeller speeds of $1,600,1,800$, and $2,000 \mathrm{rpm}$ also resulted in straight lines. This suggests that the deviations from the model observed in the case of $1.0 \%$ dodecane at 1,000 rpm (Figure 8) is minimized as a result of dispersion becoming dominant over coalescence at higher impeller speeds.

\section{Effect of B lock Copolymer Concentration and Type}

D roplet-size distribution studies in the presence of block copolymers, carried out to investigate the effect of surfactants on the dispersion kinetics of dodecane, are discussed in the following paragraphs.

Addition of L-64 increased the dispersion of the oil droplets, resulting in various regions with different dispersion characteristics. The location of these regions along the time 


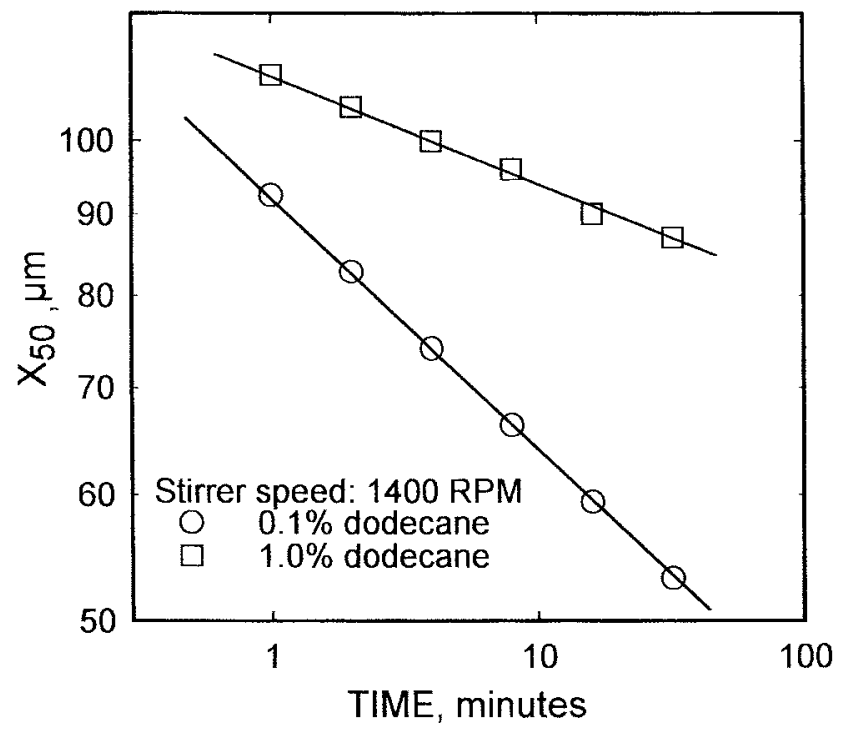

Figure 9. Change in the median droplet size as a function of time at dodecane concentrations of $0.1 \%$ and $1.0 \%$ by volume (from Polat and Chander, 1992).

axis was a function of surfactant concentration. The results are presented in Figure 10. A nother type of block copolymer with a higher molecular weight, P-104, was also tested in this group, and the same type of general behavior was observed. However, the final droplet size obtained for the same concentration was smaller in the case of P-104 due to the larger reductions in the oil/water interfacial tension observed with this reagent compared to $L-64$.

A conceptual model based on these results is presented in Figure 11 to explain the change observed in the rate of dispersion with the block copolymers. A ccording to this model,

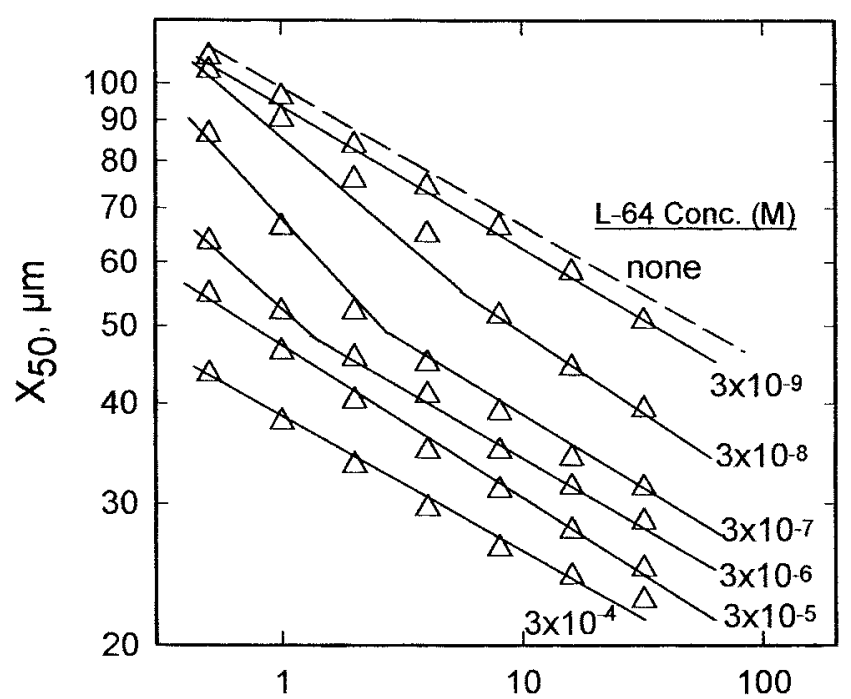

Figure 10. Effect of concentration of Pluronic L-64 on the kinetics of dispersion of dodecane.

A mount of dodecane: $0.1 \%$ by volume.

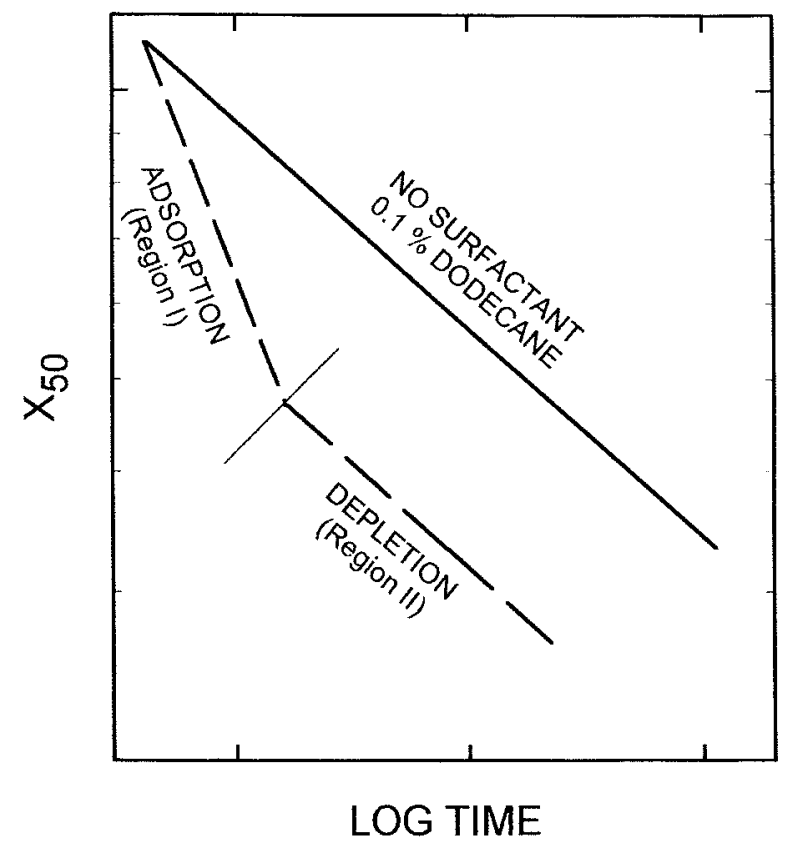

Figure 11. Effect of the surfactants on the kinetics of dispersion of dodecane.

dispersion of dodecane in the presence of surfactants can be divided into two consequent regions along the time scale.

- Region I. Characterized by large numbers of free surfactant molecules in solution. The interfacial tension is reduced by the adsorption of these free molecules at the oil/water interface, resulting in an increase in the breakage rate, and hence a higher slope in this region.

- Region II. Characterized by the depletion of surfactant molecules from the solution. Also, due to the decrease in the number of molecules, a reduction in the diffusion rate of the surfactant molecules from bulk to the oil surface can be expected in this region. Under these conditions, the breakage rate approaches that of the "no surfactant" case.

\section{Effect of Time of Reagent Addition}

The effect of the time of surfactant addition was investigated by introducing the block copolymer ( $L-64)$ into the emulsion at different times during dispersion: (1) simultaneously with dodecane; (2) after 4 min of dispersion; and (3) after 84 min of dispersion (Figure 12). E ven though the time of addition resulted in a significant difference in the kinetics of dispersion, the final state of the system was the same whether the surfactant was added simultaneously with oil or after the oil was emulsified. The observation that the droplet-size distributions became identical at long times implies that the droplets have similar final surface coverage, and supports the hypothesis that the surfactant is depleted from solution after a sufficient dispersion time.

\section{Conclusion}

This study was carried out to investigate the kinetics of dispersion in lean oil-in-water systems. A phenomenological 


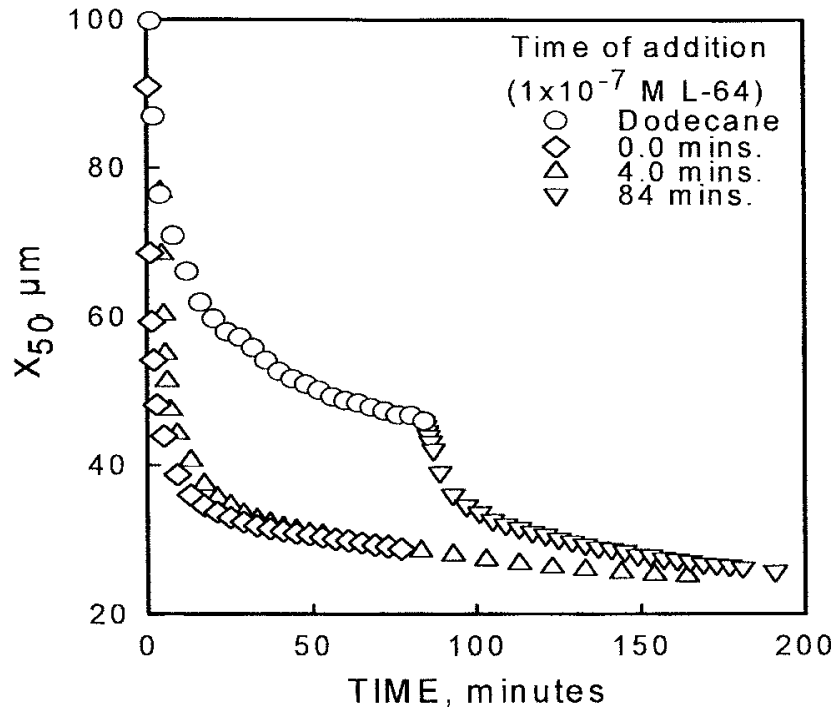

Figure 12. Effect of the time of surfactant addition on dispersion of $0.1 \%$ by volume.

model was proposed to describe the kinetics of drop breakup in the dispersion-dominated region. In addition, the effect of adding block copolymeric surfactants on the kinetics of oil dispersion was also investigated. The following conclusions were drawn:

1. The proposed model describes the dispersion behavior of oil by two empirical parameters, namely, the median droplet size at one minute of dispersion, $X_{50}(1)$, and a breakage rate constant, $k$. Expressing the dispersion data using two distinct parameters is of significant value in the evaluation of the process kinetics.

2. The model represents the dispersion of lean oil-in-water dispersions ( $>0.1 \%$ by volume) quite well for a stirrer speed of $1,000 \mathrm{rpm}$. Deviations from the model were apparent at high oil concentrations, most probably due to the increasing contribution of coalescence.

3. Increasing the impeller speed increased the ability of the model to represent the data obtained at higher oil concentrations ( $1.0 \%$ by volume).

4. Addition of block copolymers was seen to enhance the oil dispersion at all the concentrations tested. This effect was a function of surfactant type and concentration. At a fixed concentration, the drop size obtained was smaller for the more surface-active molecules.

5. Dispersion of dodecane in the presence of the surfactants seems to take place in two consequent regions along the time scale. The first region is characterized by a large number of free surfactant molecules in solution. The interfacial tension is reduced by the adsorption of these free molecules at the oil/water interface, resulting in an increase in the breakage rate. The second region is characterized by the depletion of surfactant molecules from solution and by a reduction in the diffusion rate of the surfactant molecules from bulk to the oil surface. U nder these conditions, the breakage rate decreases and approaches that of the "no surfactant" case.
6. The kinetics of dispersion changed considerably when the block copolymer was added at different times during dispersion. However, the final droplet-size distribution in the system was independent of the time the reagent was added.

\section{Acknowledgments}

The authors acknowledge the support from the $M$ ineral Processing Section, The Pennsylvania State U niversity, DOE /DOD Cooperative A greement No: DE-FC22-92PC 92162, and DOE M icro-A gglomeration No: DE-FG 22-92PC 92543.

\section{Literature Cited}

A damson, A. W., Physical Chemistry of Surfaces, Wiley, New Y ork (1990).

A rai, K., M. Konno, Y. M atunga, and S. Satio, "E ffect of D ispersed Phase $V$ iscosity on the M aximum Stable D rop Size for Break-up in Turbulent Flow," J. Chem. Eng. Jpn., 10, 325 (1977).

Becher, P., and M.J. McCann, "The Process of Emsulsification: Computer M odel," L angmuir, 7, 1325 (1991).

Calderbank, P. H., "Physical R ate Processes in Industrial Fermentation: I. The Interfacial A rea in Gas-Liquid Contacting with $\mathrm{Me}$ chanical A gitation,"'Trans. Inst. Chem. Eng., 36, 443 (1958).

Chander, S., H. Polat, and B. R. Mohal, "Flotation and Wettability of a Low-Rank Coal in the Presence of Surfactants," J. Miner. M etall. Process., 11, 55 (1994).

Coulaloglou, C. A., and L. L. Tavlarides, “D rop Size Distributions and Coalescence F requencies of $L$ iquid-L iquid $D$ ispersions in F low V essels," AlChE J., 22, 289 (1976)

Glasgow, L. A., and J. P. H su, Flocculation Sedimentation and Consolidation, B. M. M oudgil and P. Somasundaran, eds., Proceedings of the Engineering Foundation Conference, Sea I sland, GA (1985).

H iemenz, P. C., Principles of Colloid and Surface Chemistry, Dekker, New York (1985).

Hinze, J. O., "Fundamentals of the Hydrodynamic M echanism of Splitting up in Dispersion Process," AlChE J., 1, 289 (1955).

Holland, F. A., and F. S. Chapman, Liquid M ixing and Processing in Stirred Tanks, R einhold, New Y ork (1966).

Kolmogorov, A. N., Dokl. Akad. Nauk SSSR, 66, 825 (1949).

Konno, M., K. A rai, and S. Saito, "The Effect of Stabilizer on Coalescence of Dispersed Drops in Suspension Polymerization of Styrene," J. Chem. Eng. Jpn., 15, 131 (1982).

Koshy, A., T. R. Das, and R. Kumar, "E ffect of Surfactants on D rop Breakage in Turbulent Liquid Dispersions," Chem. Eng. Sci., 43, 649 (1988).

Lachaise, L., C. M endiboure, C. Dicharry, G. M arion, M. Bourrel, P. Cheneviere, and J. L. Salager, "A Simulation of E msulsification by Turbulent Stirring," J. Colloids Surf. A: Physicochem. Eng. Aspects, 94, 189 (1995).

Lagisetty, J. S., P. K. Das, R. Kumar, and K. S. G andhi, "Breakage of Viscous and Non-Newtonian Drops in Stirred Dispersions," Chem. Eng. Sci., 41, 65 (1986).

Madden, A. J., and B. M cCoy, Chem. Eng. Sci., 19, 506 (1964).

Mlynek, Y., and W. Resnick, "Drop Sizes in an A gitated LiquidLiquid System," AIChE J., 18, 122 (1972).

Narsimhan, G., D. Ramkrishna, and J. P. G upta, "A nalysis of D rop Size Distribution in Lean Liquid-Liquid Dispersions," AIChE J., 26, 99 (1980).

Nishikawa, M., F. M ori, T. Kayama, and S. Nishioka, "D rop Size Distribution in a Liquid-Liquid Phase Mixing Vessel," J. Chem. Eng. Jpn., 24, 88 (1990).

Nikolov, A. D., and D. T. W asan, "Ordered M icelle Structuring in Thin Films Formed from A nionic Surfactant Solutions," J. Colloid Interface Sci., 133, 1 (1989).

Polat, M., and S. Chander, "Kinetics of Emulsification in the Presence of Fine Particles," Dispersion and Aggregation: Fundamentals and Applications, B. M. M oudgil and P. Somasundaran, eds., Engineering Foundation, N ew Y ork (1992).

Polat, H., "The U se of PEO/PPO Tri Block Copolymers in Coal Cleaning by Flotation," PhD Thesis, Pennsylvania State Univ., U niversity Park (1995). 
Polat, H., and S. Chander, "Adsorption of PEO/PPO Triblock Copolymers and Wetting of Coal," Colloids Surf. A: Physicochem. Eng. Aspects, 146, 199 (1999).

Sachs, J. P., and J. H. R ushton, Chem. Eng. Progress, 50, 597 (1954).

Shinnar, R ., "On the Behavior of Liquid Dispersions in M ixing V essels," Fluid Mech., 10, 259 (1961)

Sleicher, C. A., "M aximum Stable Drop Size in Turbulent Flow," AIChE J., 8, 471 (1962).

Sprow, F. B., "Distribution of Drop Sizes Produced in Turbulent Liquid-Liquid Dispersion," Chem. Eng. Sci., 22, 435 (1967).

Tatterson, G. B., Fluid Mixing and Gas Dispersion in Agitated Tanks, M cG raw-Hill, N ew Y ork (1991).
Tjaberinga, W. J ., A. Boon, and A. K. Chesters, "M odel Experiments and Numerical Simulations on Emulsification Under Turbulent Conditions," Chem. Eng. Sci., 48, 285 (1993).

Vermeulen, T., G. M. Williams, and G. E. Langlois, "Interfacial A rea in Liquid-L iquid and Gas-L iquid A gitation," Chem. Eng. Progress, 51, 85F (1955).

Walstra, P., "Formation of Emulsions," Encyclopedia of Emulsion Technology, P. Becher, ed., V ol. 1, D ekker, N ew Y ork (1983).

M anuscript received Jan. 28, 1999, and revision received A pr. 26, 1999. 